\title{
Mathématiques et langage poétique chez Gilberto Owen
}

\author{
Belén Hernández Marzal
}

\section{Résumé}

On pense souvent que les mathématiques sont déconnectées du réel, mais plus encore de la poésie. Pourtant, mathématiques et poésie n'ont toujours pas été deux univers complètement étrangers. Ainsi, pour les Grecs, mathématiques et poésie faisaient partie des arts libéraux. Poésie, musique et mathématique intégraient le Quadrivium dans les études médiévales. Pour Pythagore, la musique et les mathématiques étaient des disciplines sœurs, cellesci étant inséparables de la poésie. On dit souvent, également, que les Mathématiques sont la poésie de la science. Ce n'est pas étonnant, alors, que Gilberto Owen, poète mexicain, se serve du signe mathématique en tant que signe poétique. II recourt également aux Mathématiques pour définir l'essence de la poésie : la poésie comme une énigme que le lecteur doit résoudre, d'après Owen, à la façon d'un détective, mais aussi d'un mathématicien. L'emploi de chiffres ou d'autres signes mathématiques contribue au renouvellement du langage poétique. En définitive, mathématiques et poésie se fécondent mutuellement pour créer une expression poétique singulière.

Mots-clés: mathématiques, poésie, Gilberto Owen, Mexique, symbolisme, fécondation mutuelle

\begin{abstract}
We often think that mathematics is disconnected from reality, but still more from poetry. However, mathematics and poetry have not always been two completely foreign universes. Thus, for Ancient Greeks, mathematics and poetry were part of the liberal arts. Poetry, music and mathematical were incorporated in Quadrivium in medieval studies. For Pythagoras, music and mathematics were sisters, and inseparable from poetry. It is often said, too, that mathematics is the poetry of science. This is not surprising, then, that the Mexican poet Gilberto Owen used mathematical symbols as poetic symbols. He also resorted to mathematics to define the essence of poetry: poetry as a puzzle for the reader to solve, according to Owen, as a detective, and as a mathematician too. Figures or other mathematical symbols contribute to the renewal of poetic language. Ultimately, mathematics and poetry are mutually enriching to create a singular poetic expression.
\end{abstract}

Keywords: mathematics, poetry, Gilberto Owen, Mexico, symbolism, mutual begetting 
Les mathématiques ont une présence massive dans la poésie du poète mexicain Gilberto Owen ${ }^{1}$, dépassant de loin d'autres disciplines scientifiques présentes également dans son œuvre. Soit par petites touches, par des allusions ponctuelles à des mathématiciens ou à des théories mathématiques ou par des jeux linguistico-mathématiques, le poète les intègre au corps du poème et en fait un matériau poétique. Loin d'être incongrue, cette présence met en évidence les liens entre ces deux disciplines, mathématiques et poésie, que tout oppose en principe. Nous voudrions donc montrer la part mathématique qui existe dans la poésie du Mexicain et les interactions entre mathématiques et poésie.

Pour présenter de façon schématique, on pourrait affirmer que la présence des mathématiques chez Owen obéit à deux facteurs :

- Un désir de renouveau dans le langage poétique, dans la lignée d'auteurs comme Lautréamont et ses Chants de Maldoror.

- Le souhait de renouer avec ce qu'il considère comme les origines sacrées de la poésie, époque où mathématiques, poésie et musique ne faisaient qu'un.

Gilberto Owen et les camarades de son groupe emploient deux comparaisons pour capter l'essence de la poésie, tendant ainsi des ponts avec d'autres disciplines :

Pour Jorge Cuesta, comme le signale Elías Nandino, la poésie est « un problema de multiplicación que el lector debía resolver $»^{2}$.

La deuxième, de Gilberto Owen, conjugue poésie et musique, poésie et roman policier, rapprochant par la même occasion le mystère poétique d'une énigme mathématique ou d'une équation : «Porque la neuma ${ }^{3}$ poesía, en realidad, no viene a ser sino una novela de misterio en la cual se nos dan todos los datos, pero se nos deja a cada cual encontrar la propia solución $»^{1}$.

${ }^{1}$ Rosario, Mexique, 1904-Philadelphie, Etats-Unis, 1952.

${ }^{2}$ Elías Nandino, cité par Annick Allaigre-Duny, Jorge Cuesta. Littérature, histoire, psychanalyse, p. 267.

${ }^{3}$ Le Littré donne la définition suivante du terme «neume », l'équivalent français de « neuma » : «1.Terme de plain-chant. Courte mélodie, qui est une sorte de récapitulation du mode dans lequel on vient de chanter, et qui se vocalise, sans paroles ou sur la dernière syllabe du dernier mot, à la fin des antiennes. Dans les églises où il y a des orgues, on touche de cet instrument aux fêtes annuelles et solennelles, soit majeures, soit mineures, au lieu de la neume, L'Abbé Lebeuf, Traité sur le chant ecclés. p. 242 [...] »

2. « S. m. pl. On désigne ainsi aujourd'hui les signes qui servaient au commencement du moyen âge (sic) à noter le plain-chant, et qu'on a employés, d'abord seuls, puis échafaudés sur et entre les lignes de la portée. Les notes actuelles du plain-chant ne sont, en réalité, que ces mêmes neumes, transformés, simplifiés et dénaturés par un long usage. Les signes de la notation en neumes étaient très nombreux et pouvaient varier en quelque sorte au gré de chaque copiste, suivant sa manière de grouper les sons et de lier les signes, F. Danjou, Revue de musique relig. popul. et 
On dit souvent que les mathématiciens sont un peu poètes. "Un mathématicien qui n'a pas aussi une part de poète, ne deviendra jamais un mathématicien accompli ${ }^{2}$, affirme Karl Weierstrass. Selon Sofia Vasilyevna Kovalevskaïa, disciple du mathématicien allemand, "II n'est pas possible d'être mathématicien sans avoir l'âme d'un poète $»^{3}$.Nous voudrions montrer que, parfois, les poètes sont eux aussi un peu mathématiciens.

\section{Les sciences, les mathématiques et la poésie}

Bien que les liens entre poésie et mathématiques remontent à l'Antiquité, nous allons dans un premier temps nous référer à l'œuvre de Lautréamont ${ }^{4}$, qui constitue un bon exemple de l'irruption des mathématiques -mais aussi des sciences- dans la poésie. Les séries de beau comme de cet auteur vénéré par les Surréalistes ont opéré une véritable révolution poétique en introduisant le vocabulaire scientifique en poésie. Citons en exemple la célèbre « rencontre fortuite sur une table de dissection d'une machine à coudre et d'un parapluie ! ${ }^{5}$. Mais, parmi toutes les sciences, ce sont plutôt les mathématiquesqui représenteraient pour Lautréamont la perfection absolue, érigées au rang de divinité dans cette ode aux mathématiques :

"O mathématiques sévères, je ne vous ai pas oubliées, depuis que vos savantes leçons, plus douces que le miel, filtrèrent dans mon cœur, comme une onde rafraîchissante. J'aspirais instinctivement, dès le berceau, à boire à votre source, plus ancienne que le soleil, et je continue encore de fouler le parvis sacré de votre temple solennel, moi, le plus fidèle de vos initiés " ${ }^{6}$.

classique, numéro d'août 1847. Le mot neume est parfois, mais rarement, employé au singulier dans le sens dont il s'agit ici ». Définition du Littré, entrée « Neume »,

http://francois.gannaz.free.fr/Littre/xmlittre.php?requete=neume\&submit=Rechercher

1 «Car la neume poésie n'est, en réalité, qu'un roman de mystère dans lequel on nous fournit tous les éléments mais on laisse à chacun le soin de trouver sa propre solution », in Gilberto Owen, Obras, p. 288.

${ }^{2}$ Arild Stubhaug, Sophus Lie : une pensée audacieuse, p. 100.

${ }^{3}$ http://www.apprendre-en-ligne.net/blog/index.php/Citations/p7

${ }^{4}$ Montevideo 1846-Paris 1870.

${ }^{5}$ Voici la citation in extenso : «Il est beau comme la rétractilité des serres des oiseaux rapaces; ou encore, comme l'incertitude des mouvements musculaires dans les plaies des parties molles de la région cervicale postérieures ; ou plutôt, comme ce piège à rats perpétuel, toujours retendu par l'animal pris, qui peut prendre seul des rongeurs indéfiniment, et fonctionner même caché sous la paille; et surtout, comme la rencontre fortuite sur une table de dissection d'une machine à coudre et d'un parapluie! ", Ducasse, Les Chants de Maldoror, p. 233-234.

${ }^{6}$ Ducasse, Chant deuxième, ibid., p. 89. Norbert Meusnier propose une analyse de l'ode aux mathématiques ducassienne dans «Isidore Ducasse, géomètre de la poésie ». 
II ne tarit pas d'éloges au sujet des mathématiques et de ses différentes branches : « 0 Mathématiques saintes, puissiez-vous, par votre commerce perpétuel, consoler le reste de mes jours de la méchanceté de l'homme et de l'injustice du Grand-tout ! " ${ }^{1}$; Arithmétique ! algèbre ! géométrie! trinité grandiose! triangle lumineux! Celui qui ne vous a pas connues est un insensé ! $»^{2}$. Au milieu de ce cri de révolte que sont Les Chants de Maldoror, les mathématiques apparaissent comme un refuge : "Et au milieu des chants de révolte s'épanouit, comme une oasis, l'hymne aux mathématiques, les divinités souveraines, recours et consolation du révolté ${ }^{3}$. En effet, elles constituent pour Lautréamont un exemple d'ordre et de rigueur, qualités qu'il voudrait pour sa poésie, très influencé qu'il était par le milieu hispano-américain, fortement imprégné de positivisme, qu'il aurait fréquenté à Paris ${ }^{4}$.

L'héritage positiviste a laissé aussi ses traces sur l'éducation de Gilberto Owen, qui poursuit ses études à la Escuela Nacional Preparatoria ${ }^{5}$, fondée par Gabino Barreda ${ }^{6}$, introducteur introducteur du positivisme et des idées comtiennes au Mexique. Cette école, dont le slogan était "Amour, ordre et progrès ", a formé des gens comme Frida Kahlo?.

${ }^{1}$ lbid., p. 94.

${ }^{2}$ Ibid., p. 90.

${ }^{3}$ Suzanne Bernard, Le poème en prose, de Baudelaire jusqu'à nos jours, p. 222.

${ }^{4}$ L'intelligentsia hispano-américaine à Paris prônait les « idées de progrès et de démocratie [...] convaincue que l'avenir est à l'union de la littérature et des sciences, au fédéralisme, à l'éducation populaire ", Michel Pierssens, Ducasse et Lautréamont : L'envers et l'endroit, p. 37. La thèse de Michel Pierssens est que Lautréamont serait un auteur latino-américain à Paris, d'où les nombreux hispanismes dans les Chants de Maldoror: «[...] c'est à une seconde hypothèse que je vais consacrer la suite de cet exposé. En deux mots la voici : Ducasse n'était pas un écrivain français mais un littérateur hispano-américain de Paris [...]. Si l'on en croit Guy Laflèche, les hispanismes seraient même à ce point répandus dans les Chants qu'il faudrait supposer que ces derniers ont été pensés en espagnol avant d'être rédigés en français ", ibid., p. 31. Enfin, l'auteur énonce l'hypothèse suivante : "si nous n'avons encore trouvé que de rares traces de Ducasse dans les milieux littéraires parisiens de son temps, c'est parce qu'il fréquentait avant tout ses véritables pairs : les hispano-américains de Paris » Pierssens, idem.

${ }^{5}$ Inés Arredondo, « Apuntes para una biografía», p. 44.

${ }^{6} 1818-1881$.

${ }^{7}$ La formation scientifique dispensée par l’Ecole Préparatoire se caractérise par son excellence et sa solidité : «L'enseignement délivré dans cet établissement relevait davantage du supérieur que du secondaire. Gabino Barreda, son premier directeur, comparait le programme à une échelle du savoir dont le premier degré était occupé par les mathématiques et le dernier par la logique. Entre ces deux extrêmes, les étudiants recevaient une solide formation de physique et de biologie. L'apprentissage des langues était lié à celui des sciences: il débutait par le français, se poursuivait par l'anglais, parfois par l'allemand, et s'achevait, les deux dernières années, par le latin. "Voici notre devise : liberté, ordre et progrès ; la liberté sera notre moyen, l’ordre notre assise et le progrès notre but", déclarait 


\section{Les découvertes scientifiques dans la poésie du $\mathrm{XX}^{\mathrm{e}}$ siècle : mathématiques et $4^{\mathrm{e}}$ dimension.}

La « contamination » de la poésie par les sciences gagne du terrain au $X X^{e}$ siècle. Loin de se limiter à la poésie, elle s'étend à tous les domaines des arts. Ainsi, la découverte de la quatrième dimension a bouleversé nos représentations poétiques et picturales. L'on pourrait se demander, avec Suzanne Bernard, s'il ne faudrait pas voir dans la quatrième dimension :

"[U]n effort mené par chaque art pour sortir des catégories où on l'avait jusqu'alors enfermé, pour acquérir la dimension qui lui manque : la peinture, art de l'espace, s'annexant le temps; la poésie, art du temps, s'annexant l'espace. On peut penser en effet que la peinture cubiste se propose de représenter sur la toile à deux dimensions non seulement la troisième dimension (le volume des objets) mais en même temps la "quatrième dimension", c'est-à-dire la durée : pourquoi, écrivent Gleizes et Metzinger, l'esprit ne tournerait pas " autour de l'objet pour en saisir plusieurs apparences successives qui, fondues en une seule image, le reconstituent dans la durée ?". $C^{\prime}$ est ainsi que les cubistes représentent simultanément sur la toile les aspects multiples d'un même objet, vus sous différents angles. Inversement la poésie, art du temps -puisque liée, pensait-on jusqu'alors, à une lecture forcément successive- va s'efforcer d'emprunter à la peinture son caractère spatial et simultané. Je n'insisterai pas sur l'aspect métaphysique de telles ambitions, dont on peut dire qu'elles visent à transgresser les lois apparentes du réel pour mieux appréhender ce réel dans sa totalité; mais il est intéressant de voir en quoi elles ont pu atteindre et modifier les structures littéraires ${ }^{1}$.

Ainsi, les artistes, très influencés par les théories d'Einstein et l'ouvrage La science et l'hypothèse de Poincaré, ont appliqué ces théories au domaine des arts, le cubisme en constitue le meilleur exemple. Les demoisellesd'Avignon de Picasso,première toile cubiste, datent de 1907, seulement deux ans après la première ébauche de la théorie de la relativité. Owen, lui, est influencé par le cubisme, qui n'est que la tentative picturale pour traduire la quatrième dimension ${ }^{2}$, et nous trouvons des traces de ces théories dans ses textes. En effet, lorsqu'il est

Barreda, réinterprétant ainsi la maxime gravée dans la pierre du blason de la Preparatoria : Amour, Ordreet Progrès ", Hayden Herrera, Frida, Biographie de Frida Kahlo, p. 43.

${ }^{1}$ Bernard, Le poème en prose, de Baudelaire jusqu'à nos jours, p. 619-620.

${ }^{2}$ «Lo mejor es tenderse, cruzados los brazos, ante el rompecabezas plástico de ese rostro descompuesto, como por el olvido, por la lente poliédrica del botellón, allí enfrente. La nariz, bajo la boca, en el lugar del cuello. Tiene, aislada, un valor definitorio independiente; sensual, nerviosa, de aletas eléctricas como carne de rana en un experimento de laboratorio. Dos pares de ojos, en el lugar de las orejas, le brillan como dos aretes líquidos, 
question dans sa poésie d'un «espacio de sólo tres dimensiones ${ }^{1}$, ce qui, de prime abord, pourrait avoir l'air d'une banale référence à la géométrie classique suggère l'existence de la quatrième dimension, c'est-à-dire la notion d'espace-temps introduite en physique depuis la théorie de la relativité d'Einstein. C'est la restriction introduite par l'adverbe sólo qui révèle sa connaissance de la quatrième dimension einsteinienne. De même, il est question dans le poème "Acróstico » de la dimension temporelle, devenue ici, par élimination de l'une des dimensions, la troisième dimension : « Y hay la mirada fría y plana del acero / que nos unta a su espejo sin amor / $Y$ cuando salimos tenemos tres dimensiones / Pero la tercera es el tiempo no más ${ }^{2}$.Dans les deux descriptions, la quatrième dimension, c'est-à-dire l'espace-temps, remplace l'une des dimensions classiques. L'espace représenté dans ces poèmes pâtit de cette suppression et se révèle insuffisant et bancal. L'introduction du miroir explique en partie ce phénomène d'élimination, car il efface automatiquement l'une des dimensions, à savoir la profondeur.

II ne fait plus de doute qu'Owen était au courant des recherches de Poincaré et de ses conjectures au sujet de la quatrième dimension dès lors que le scientifique devient un personnage du poème "Repeticiones » : " [...] empezó a oír a un Poincaré matemático que le llevaba con muy suaves modales a que el fin de la vida era la contemplación $»^{3}$ (p. 108). Mais sa vision est celle d'un poète et non d'un mathématicien, et le Poincaré d'Owen, plongé dans la contemplation, est plus proche d'un mystique que d'un scientifique. Et ce sont sans doute les implications métaphysiques de la théorie de la $4^{\mathrm{e}}$ dimension qui intéressent le poète Owen.

incendiados. Así serían las joyas de la corona, hechas con los ojos coléricos de los mujiks rebeldes. La frente es todo el resto de la cara, multiplicada su convexidad por la del cristal de la botella », "Le mieux c'est de s'allonger, les bras croisés, devant le puzzle plastique de ce visage décomposé, comme par l’oubli, par la lentille polyédrique de la bouteille, là devant. Le nez, sous la bouche, à la place du cou. Elle a, isolée, une valeur définitoire indépendante ; sensuelle, nerveuse, les ailes électriques comme de la chair de grenouille dans une expérience de laboratoire. Deux paires d'yeux, à la place des oreilles, brillent comme des boucles d'oreille liquides, incendiés. C'est comme cela qu'elles doivent être, les joyaux de la couronne, réalisés avec les yeux coléreux des Moujiks rebelles. Le front occupe toute le reste du visage, multipliée sa convexité par celle du verre de la bouteille », Owen, Obras, p. 149.

1 « Espace à trois dimensions seulement ».

${ }^{2}$ « II y a le regard froid et plat de l'acier / qui nous frotte à son miroir sans amour / Et lorsque nous sortons nous avons trois dimensions / Mais la troisième est seulement le temps ». Dans le poème "El río sin tacto ", Owen utilise une image très proche en ce que, comme dans "Acróstico ", il procède à l'élimination d'une des dimensions : «Se le veía mal por sus tres dimensiones mediterráneas. Había que sustituir una por el tiempo. Había que eliminar una. Prefirió adelgazar. Se volvió largo y profundo, sin frente », (« On ne le voyait pas bien à cause de ses trois dimensions méditerranéennes. Il fallait en remplacer une par le temps. Il fallait en éliminer une. II préféra maigrir. II devint long et profond, sans front $»)$, Ibid., p. 109, c'est nous qui soulignons.

${ }^{3}$ Ibid., p. 108.

R. B. E. C. T., vol 6, núm. 1, jan-abr.2013 ISSN - 1982-873X

Artigo apresentado na Premières Journées d'études organisées à Lyon (France) 


\title{
Mathématiques et abstraction : ordre et rigueur
}

Le rapport aux mathématiques est plutôt ambivalent chez Owen. La référence au nombre permet d'abord à Owen de répertorier les objets et par là même de rendre compte du monde qui l'entoure, d'où leur présence dans ses poèmes et dans sa correspondance. Le nombre s'avère alors une présence rassurante, chargé celui-ci d'instaurer l'ordre et la rigueur. Mais le nombre peut aussi être perçu comme une présence menaçante, car il ôte aux êtres toute partde mystère. Par ailleurs, définir une personne en quelques chiffres relève plutôt du travail du bureaucrate, des fiches que rédigeait Owen dans son bureau du consulat : "Los números me amenazan. Si los oigo, sabré todo lo de tu vida, tus años, tus pestañas, tus dedos, todo lo que ahora cae, inmóvil, como en las grutas, espacio de sólo tres dimensiones $»^{1}$.

En outre, les nombres et les mathématiques n'étant au fond que pure abstraction, la première impression d'objectivité et de réalité s'avère illusoire. Le nombre réduit l'être à la virtualité, le déconnectant du réel. Ainsi, si dans un premier temps il a trouvé refuge dans la présence rassurante du nombre-dans un premier temps, les statistiques peuvent donner à Owen l'illusion de mieux comprendre la grande et insaisissable ville qu'est New York-il finit par réaliser que le nombre a quelque chose d'inhumain :

\begin{abstract}
"A New York se la empieza a ver desde el subway. Acaba allí la perspectiva plana, horizontal. Empieza un paisaje de bulto ahí, con la doble profundidad, o eso que llaman cuarta dimensión, del tiempo. Es mucho más fácil entenderlo, claro está, desde la estadística.Pero la pureza inhumana del número es otra exageración peligrosa. Al principio me refugié en ella. Conservo unas notas: The rapid transit companies of the city carry a total of about 1880000000 people a year $"^{2}$.
\end{abstract}

Il en est encore question, dans ce poème, de la quatrième dimension. Il est intéressant de constater à quel point Owen intègre des notions mathématiques ou scientifiques dans la

\footnotetext{
${ }^{1}$ « Les nombres me menacent. Si je les écoute je saurai tout sur ta vie, ton âge, tes cils, tes doigts, tout ce qui tombe maintenant, immobile, comme dans les grottes, espace de seulement trois dimensions ", Ibid., p. 62.

${ }^{2}$ « On commence à voir New York depuis le subway. C'est là que finit la perspective plate, horizontale. C'est là que commence un paysage volumineux, avec la double profondeur, ou ce qu'on appelle quatrième dimension, celle du temps. C'est plus facil à comprendre, bien entendu, depuis les statistiques. Mais la pureté inhumaine du nombre est une autre exagération dangereuse. Je me suis réfugié au début chez elle. Je conserve quelques notes: The rapid transit companies of the city carry a total of about 1880000000 people a year (Extrait d'une lettre à Celestino Gorostiza, c'est nous qui soulignons, Ibid., p. 270).
} 
description de son propre réel, de son quotidien, au point que l'on est tenté de se demander si ces théories scientifiques ne répondent pas à ses préoccupations et interrogations intimes.

Mais le nombre, qu'il qualifie d'inhumain (" pureté inhumaine du nombre »), est aussi théorique et abstrait que fantomatique et irréel, comme il l'affirme à la fin de cette même lettre :

«Pero el número, te digo, es nomás un poema. Nada más. Una teoría de una teoría fantasma de fantasma. La realidad artística se refugia en esas figuras retóricas por pereza, por pobreza $»^{1}$.

Nombre qui conduit l'être au néant, comme dans le poème "Autorretrato o del Subway ", où les numéraux finissent par anéantir la ville : "[...] los numerales hacen hoy más esta ciudad una mera hipótesis ». Ainsi, si les chiffres permettent de produire un exposé clair, scientifique et objectif, cette impression se dissout pour ne laisser qu'une présence fantomatique. Les mathématiques apparaissent comme une construction mentale, purement hypothétique, vide et inexistante.

\section{Mathématiques et poésie}

Si pour Owen le nombre est poème, la musique l'est aussi ; c'est alors qu'il renoue avec les théories pythagoriciennes. Cette conception n'est pas nouvelle, puisque depuis Pythagore l'on a signalé les rapports entre le nombre et les arts, notamment la musique ${ }^{2}$. Basée sur les rapports numériques harmonieux, cette conception de l'harmonie est restée ancrée pendant des siècles. Owen n'ignorait pas les théories antiques sur les rapports entre les mathématiques, la poésie et les autres arts, comme le prouvent les allusions à Pythagore dans ses œuvres :

« La música. Llega de lejos. En el camino se entretiene bailando, con la horda, en torno de las hogueras ; luego se martiriza, gime y se hace salmo bíblico, o se perfuma y se

${ }^{1}$ « Mais le nombre, je te le dis, est seulement poème. Rien d'autre. Une théorie d'une théorie fantôme de fantôme. La réalité artistique se réfugie dans ces figures rhétoriques par paresse, par pauvreté »,lbid., p. 271.

${ }^{2}$ Par ailleurs, la septième acception du substantif chiffre est une acception musicale : « S. m. plur. Dans la musique, caractères numériques qu'on place au-dessus des notes de la basse pour indiquer les accords qu'elle comporte. Ainsi 5 ou 3 avec le 5 au-dessus indique l'accord de tierce et quinte, c'est-à-dire l'accord parfait majeur ; l'accord parfait mineur met un bémol à la place du 3 ».Définition du Littré. Entrée « Chiffe » :

http://francois.gannaz.free.fr/Littre/xmlittre.php?requete=chiffre\&submit=Rechercher 
ace carne de sirena. Pero Pitágoras, con su muslo de oro, vivía junto al taller de un herrero, y la hizo número » ${ }^{1}$.

II fait référence ci-dessus à la cuisse d'or que la légende attribuait à Pythagore. II est question également de la forge qui serait à l'origine de ses théories, comme il l'affirme dans l'exemple qui suit :

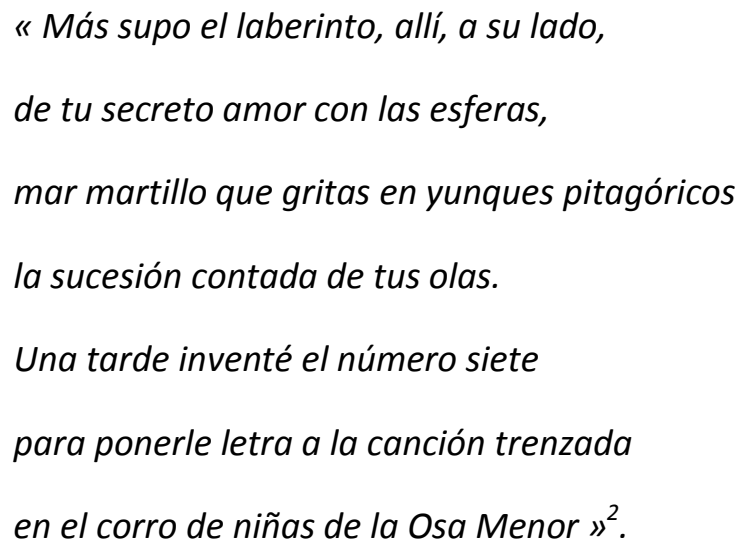

Ainsi, le mouvement des vagues de la mer semble régi par l'ordre divin, la succession des vagues rappelant l'intervalle des coups de marteau sur l'enclume, les mêmes qui auraient permis à Pythagore, lorsqu'il passait devant une forge, d'appréhender les rapports mathématiques et harmonieux entre les sons. Par ailleurs, dans ce poème, le chiffre 7 , symbole de perfection ${ }^{3}$, se substitue pour Owen à laparole, "para poner letra ", mais aussi à la musique, puisque l'une des traductions deletraserait les paroles d'une chanson. Mathématiques, poésie, et même astronomie, s'allient dans ce poème pour exprimer la perfection de la beauté.

C'est pourquoi, dans une de ses lettres, Owen met le terme poesía en apposition au substantif neuma: "Porque la neuma poesía, en realidad, no viene a ser sino una novela de

1 « La musique. Elle vient de loin. Sur son chemin, elle s'amuse en dansant, dans la horde, autour des bûchers ; puis, elle est martyrisée, gémit et devient psaume biblique, ou bien elle se parfume et devient chair de sirène. Mais Pythagore, à la cuisse d'or, habitait près de l'atelier d'un forgeron et la transforma en nombre ", Owen, Obras, p. 195.

${ }^{2}$ « Le labyrinthe, là-bas, à côté de lui, savait davantage / De ton secret amour pour les sphères / Mer-marteau qui cries sur des enclumes pythagoriciennes, / le décompte successif de tes vagues.

Un soir j'ai inventé le chiffre sept /pour écrire les paroles de la chanson tressée / dans la ronde des fillettes de la Petite Ourse ", Ibid., p. 82.

${ }^{3}$ «Sept correspond aux sept jours de la semaine, aux sept planètes, aux sept degrés de la perfection, au sept sphères ou degrés célestes, aux sept pétales de la rose [...], etc. ", Jean Chevalier et Alain Gheerbrant, Dictionnaire des symboles. Article « Sept », p. 860. 
misterio ${ }^{1}$. Ce terme, appartenant au lexique musical, signifie : « Notación que se empleaba para escribir la música antes del sistema actual $»^{2}$. C'est dire à quel point poésie et musique sont liées pour Owen.

De même, dans le poème "El llamado sándalo », Owen envisage les rapports existants entre la poésie et la musique comme des rapports de voisinage, où Lupe, la chimère-musique du poème, est voisine de Pythagore : "Yo conocí una quimera [...] La bauticé muy raro : Lupe, y era la música. En esto no entraba el corazón para nada, pues era vecina de Pitágoras $»^{3}$. Ce "voisinage », si étrange qu'il puisse paraître dans un premier temps, ne l'est pas pour autant, si l'on pense aux rapports entre le pythagorisme et la musique ${ }^{4}$. Ainsi, comme le rappelle Steiner, poésie et musique sont "si étroitement mêlées que la mythologie leur attribue une origine commune $»^{5}$. Musique et mathématiques sont si proches qu'au Moyen-Âge, ellesfaisaient partie des enseignements du Quadrivium ${ }^{6}$ :

«El carácter matemático de la música determinará su función entre las restantes disciplinas del Quadrivium. [...] Platón había descrito en la República un programa de estudios para adultos fundamentado sobre las cuatro disciplinas matemáticas:

${ }^{1}$ Owen, Obras, p. 288, Voir la traduction note 5.

${ }^{2}$ Définition du Diccionario de la Real Academia Española. Entrée «neuma», $2^{\mathrm{e}}$ tome, p. 1578.

${ }^{3}$ Owen, Obras, p. 67, « J'ai rencontré une chimère [...] Je l'ai baptisée de façon très bizarre : Lupe, et c'était la musique. Le cœur n’y était pour rien, car elle était voisine de Pythagore ».

${ }^{4}$ « Los pitagóricos fueron los primeros que desarrollaron la teoría matemática de la música. Consideraban el número como el elemento esencial de todas las cosas [...] Con el desarrollo del neoplatonismo (II-III d. de J.C.), como veremos, la música formará parte definitivamente de las disciplinas matemáticas », Paloma Otaola González, " En torno a la música y las artes liberales», p. 148. ("Les Pythagoriciens ont été les premiers à développer la théorie mathématique de la musique. Ils considéraient le nombre comme le fondement essentiel de toute chose [...]. Avec le développement du néoplatonisme $\left(\mathrm{II}^{\mathrm{e}}-\mathrm{III} \mathrm{e}\right.$ av. J.-C.), comme nous le verrons, la musique fera définitivement partie des disciplines mathématiques $»)$.

${ }^{5}$ Cité par Annette de la Motte, Au-delà du mot. Une "écriture du silence » dans la littérature française au vingtième siècle, p. 50 .

${ }^{6}$ « Au Moyen-Age, le programme d'études se composait de deux cycles, le Trivium et le Quadrivium. Le Quadrivium, constitué par les quatre sciences dites mathématiques : arithmétique, musique, géométrie et astronomie et le Trivium, ou les trois sciences du langage : grammaire, rhétorique et dialectique ». Paloma Otaola González, ibid., p.148-149.

R. B. E. C. T., vol 6, núm. 1, jan-abr.2013 ISSN - 1982-873X 
aritmética, música, geometría y astronomía. Las cuatro ciencias se encuentran íntimamente ligadas entre sí por un elemento común : el número » .

Enfin, Owen rejoint les théories pythagoriciennes puisqu'il assimile le nombre au poème, au même titre que la musique, devenue nombre grâce à Pythagore ${ }^{2}$ : « Pero el número, te digo, es nomás un poema ${ }^{3}$.Nombre et poésie étant donc interchangeables,le mystère de la poésie peut être rendu à l'aide des chiffres romains dans cette strophe qui s'apparente à un véritable rébus :

" Les dice : "El tiempo es una voz

hallada entre segundos como sílabas,

que si es poesía escribirás con equis

y si es su conciencia se ha de llamar en números romanos quince;

tiene los doce pétalos de rosa de la escala, y es el trébol feliz de cuatro hojas que forma las praderas y sus distancias y sus estaciones" [...] ${ }^{4}$.

Et c'est ici que nous percevons le mieux les interactions entre mathématiques et poésie chez Owen. En effet, si le nombre est poème, le chiffre peut être lettre. Ainsi le nombre quinze, écrit en chiffres romains (XV), désigne les initiales de Xavier Villaurrutia, ami poète, conférant au chiffre un double statut (nombre et lettre). Nous en déduisons que Xavier Villaurrutia constituepour lui la personnification de la poésie, d'autant qu'il est associé à la rose, liée celle-ci à la fois à la poésie et à Villaurrutia puisqu'elle comporte autant depétales que de lettres contient son nom, c'est-à-dire douze. II faudrait signaler également le double sens du complément de nom " de la escala », qui fait référence à l'échelle, mais aussi au sens musical de ce terme, c'est-à-dire à la gamme. Le poète crée ainsi un code secret, le chiffre romain véhicule de ce fait un message chiffré, actualisant donc deux des acceptions du substantif chiffre : « entrelacement des initiales »

1 «Le caractère mathématique de la musique déterminera sa fonction parmi les autres disciplines du Quadrivium [...]. Platon avait décrit dans la République un programme d'études pour adultes basé sur les quatre disciplines mathématiques: arithmétique, musique, géométrie et astronomie. Les quatre sciences sont intimement liées entre elles par un élément commun : le nombre ». Ibid., p. 148.

2 «Pero Pitágoras, con su muslo de oro, vivía junto al taller de un herrero, y la hizo número » (« Mais Pythagore, à la cuisse d'or, vivait près de l'atelier d'un forgeron et la transforma en nombre »), p. 195.

${ }^{3}$ Ibid., p. 271.

${ }^{4}$ « Le temps est une voix / trouvée entre secondes comme des syllabes / si elle est poésie tu l'écriras avec X / et si c'est sa conscience, elle s'appellera quinze en nombres romains ; / elle a les douze pétales de rose de l'échelle » " De la ardua lección » (« De la leçon ardue »), Ibid., p. 116. 
et " [...] caractères de convention pour une correspondance secrète $»^{1}$. En définitive, la lettre devenant chiffre, le signe se transforme en message chiffré.

\section{Le caractère figuratif du chiffre. De sa réversibilité}

Si les mathématiques représentent pour Owen l'abstraction suprême, l'utilisation qu'il fait des chiffres, des nombres, est aussi très concrète, car ce qui l'intéresse avant tout c'est leur capacité à représenter, leur réversibilité. Le chiffre se donne à voir et devient dessin sur la page. Par exemple, dans son roman Novela como nube, le numéro de téléphone du personnage féminin, composé d'une succession de 2, évoque une procession de cygnes : « [...] Eva se acerca a Ernesto [...] y le entrega un carnet con su nombre, su dirección y el número de su teléfono, que es una procesión de cisnes : $2222222 \Perp^{2}$.

Si ces jeux sont assez courants en langue espagnole, où le nombre 22 est souvent appelé " los dos patitos » (les deux petits canards), Owen va plus loin dans la visualisation du nombre, le tournant et le retournant pour mieux l'adapter à ses besoins figuratifs, comme dans son poème " El estilo y el hombre » : « Mueren al unísono cisnes; alargando hacia arriba los cuellos, son más bien 7777 al revés ». L'équivalence ainsi affirmée des nombres 7777 et 2222 témoigne du fait que c'est bien le caractère figuratif du signe mathématique qui prime chez Owen. En fait, il s'agit ici de deux représentations d'un même groupe de cygnes : tout d'abord en position " normale ", 2222, puis le cou étiré, 7777 . Mais pour que la représentation soit réaliste ce dernier nombre doit être lu à l'envers. En effet, il faut retourner le chiffre 7 pour qu'il ressemble vraiment à un cygne ${ }^{3}$.

${ }^{1}$ Le Littré en ligne, $\underline{\text { http://francois.gannaz.free.fr/Littre/xmlittre.php?requete=chiffre }}$

${ }^{2}$ Owen, Obras, p. 160

${ }^{3}$ Rubén Darío, chantre du modernisme, est un précurseur de ce genre de jeux graphiques dans la poésie en langue espagnole. En effet, le poète nicaraguayen se plaisait à représenter le cygne, animal emblématique du mouvement auquel il adhérait, avec un point d'interrogation, censé représenter le cou de l'animal. En outre, il jouait de I'homophonie existant dans la langue française entre cygne et signe : « ¿ qué signo haces, cisne, con tu encorvado cuello ? [...] yo interrogo a la esfinge que el porvenir espera / con la interrogación de tu cuello divino » (Quel signe faistu, cygne, de ton cou courbé? [...] j'interroge le sphynx qui attend l'avenir / avec l'interrogation de ton col divin). Cf. le poème « Los Cisnes », in Rubén Darío, Azul... / Cantos de Vida y Esperanza, p. 212-213.

Ramón Gómez de la Serna pratique également ce genre de jeux visuels lorsqu'il combine le texte et l'illustration graphique de ses propos, et il se sert, comme Owen, du chiffre 2 pour évoquer le cygne, notamment lorsqu'il dessine un couple de cygnes pour illustrer une de ses greguerías : «Las parejas de cisnes parece que señalan siempre una misma cifra, el 22 ; pero a veces, cuando uno de ellos está entrado en el agua y el otro está en pie, a la orilla, señalan el 24 », Ramón Gómez de la Serna, Greguerías, p. 97-98. (" On dirait que les couples de cygnes forment 
De même, dans son poème "El río sin tacto ", c'est également l'iconicité du chiffre qui est mise en avant à travers les nombres 88 et $0^{\circ}$, qui évoquent pour l'un des traces de pieds nus sur le sable, et pour l'autre des traces de chaussures féminines à talon et un soleil avec sa planète. Toutefois, les possibilités expressives du chiffre ne sont pas épuisées, car les mêmes signes -les mêmes chiffres- peuvent avoir d'autres significations dans d'autres poèmes de l'auteur. En effet, preuve de la réversibilité des chiffres et des lettres chez Owen, ce même signe $-0^{\circ}-$ peut être lu comme l'initiale du nom Owen, c'est-à-dire le $O$ majuscule suivi du $O$ minuscule -le symbole " ${ }^{\circ} "$ servant à désigner les degrés- autrement dit Owen père et son fils, le petit Gilberto ${ }^{1}$. Ou, pour reprendre l'expression du poète lui-même : « contacto bobo del cero grados que era su inicial »².

Cette interchangeabilité des caractères linguistiques et mathématiques chez Gilberto Owen, qui constitue à nos yeux son originalité majeure, semble liée à l'étymologie même du substantif chiffre, qui signifiait au $X V^{\mathrm{e}}$ siècle "écriture secrète" ${ }^{3}-$; chiffre et écriture seraient étroitement liés. Et le chiffre est souvent chiffré chez Owen.

Chiffres et lettres réversibles, chiffres et messages chiffrés sont une constante dans la poésie d'Owen. En effet, le chiffre, le guarismo ${ }^{4}$, la cifra et la lettre $X$-signifiant double, à la fois chiffre romain et lettre majuscule- apparaissent très tôt déjà, dans le poème "Nueva Nao de Amor " :
"8- Guarismo que repite, interminable,
la huella de tu paso
sobre mi vida horizontal de ahora.
i Qué dulzura del viaje, enarenados
ya los caminos de la tierra,
y resuelta en tu cifra

toujours le même nombre, le 22; mais parfois, lorsque l'un d'eux est dans l'eau et l'autre debout, sur les berges, ils forment le nombre $24 »)$.

${ }^{1}$ Voir notamment l'article de Guillermo Sheridan « Gilberto Owen y el torbellino rubio », in Vuelta, $\mathrm{n}^{\circ} 239$, octubre de 1996, p. 12, http://www.letraslibres.com/pdf.php?id=4904 : «El Owen pequeño junto al Owen adulto es tema reiterado en toda la obra, sobre todo en la narrativa. Incluso en sus iniciales : Owen habla con frecuencia de los "cero grados que era tu nombre" " ("Le petit Owen aux côtés de l'Owen adulte est un sujet récurrent dans toute son oeuvre, surtout dans la narrative. Même ses initiales : Owen parle souvent des "zéro degrès qu'était ton nom" ").

${ }^{2}$ « Contact bête du zéro degrés qu'était son nom ». « El infierno perdido » (« L'enfer perdu »), p. 119.

${ }^{3}$ C'est l'étymologie que donne le Petit Robert. Entrée consultée : « chiffre », p. 305.

${ }^{4}$ Notons au passage qu'il existe l'expression letra de guarismo, dont la définition est « signo o cifra arábiga » selon le Diccionario de la Real Academia Española. Entrée " guarismo », $1^{\mathrm{er}}$ tome, p. 1171. 
la $X$ de las encrucijadas $»^{1}$.

Mais à la double signification de la lettre $X$ que nous venons de signaler s'en ajoutent encore deux autres : celle concrète, purement visuelle, du signe $X$ comme un carrefour et la signification mathématiques $d^{\prime}$ ' inconnue ». Cette lecture est possible grâce à l'utilisation du participe resuelta, car la lettre $X$ est employée dans le langage mathématique pour désigner une inconnue $^{2}$. Cet emploi de la lettre $X$ en fait un signifiant hybride, mi-mathématique, milinguistique, comme si le chiffre et la lettre se superposaient nous laissant entrevoir simultanément ses différents sens, et c'est là que réside l'originalité $d^{\prime} O{ }^{\prime}{ }^{3}$. Et le dictionnaire est encore une fois très éclairant au propos de l'ambiguïté du signe $X$, puisque la sixième acception du Littré nous apprend que la lettre $X$ désigne en langage familier les mathématiques : «Familièrement, les $x$, l'algèbre, les mathématiques. Fort en $x$, fort en mathématiques ${ }^{4}$.

Enfin, il semblerait que le substantif guarismo ${ }^{5}$ et le chiffre 8 soient équivalents dans I'univers d'Owen, ce qui explique l'apparition de ce substantif, qui n'est pourtant pas très usité en espagnol, dans le huitième poème de "Nueva nao de amor ", si l'on en croit le poème «El río sin tacto », où il appelle le chiffre 88 « los dos guarismos $»^{6}$ :

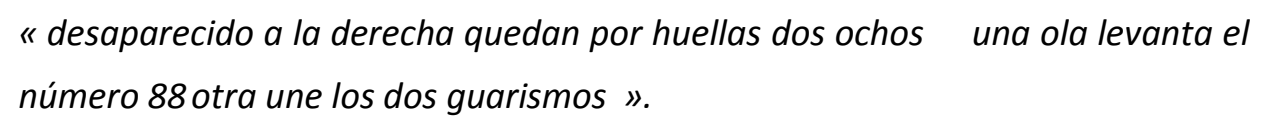

${ }^{1}$ «Chiffe qui répète, interminable, / la trace de ton pas / sur ma vie présente horizontale. / Quelle douceur que celle du voyage, ensablés / déjà les chemins par la terre, / résolu dans ton chiffre / le X des carrefours ", Owen, Obras, p. 38.

${ }^{2}$ Par ailleurs, les rayons $\mathrm{x}$ sont ainsi nommés en raison de leur caractère inconnu, car il s'agissait d'un rayonnement jusqu'alors inconnu.

${ }^{3} \mathrm{Ce}$ jeu se poursuit encore dans son roman Examen de pausas, confirmant la double lecture, entre mathématique (inconnue) : «Queremos amarte, X. -i Por quién substituir esta X ?» (p. 195), « ¿ Por quién substituir la X ? » (p. 196) (Nous voulons t'aimer, X. Par qui faut-il remplacer ce X ? », p. 195 ; «Par qui faut-il remplacer le X?», p. 196).

${ }^{4}$ Le Littré, entrée $\mathrm{x}$ », http://francois.gannaz.free.fr/Littre/xmlittre.php?rand=\&requete=x\&submit=Rechercher

${ }^{5}$ « Guarismo, ma. (Cf. algoritmo). 1. adj. ant. Perteneciente o relativo a los números. 2. m. Cada uno de los signos o cifras arábigas que expresan una cantidad. 3. m. Expresión de cantidad compuesta de dos o más cifras» (" Relatif aux chiffres. 2. m. Chacun des signes ou chiffres arabes exprimant une quantité. 3. m. Expression de quantité composé de deux ou plus chiffres »). Diccionario de la Real Academia de la Lengua, entrée "guarismo », $1^{\mathrm{er}}$ tome, $\mathrm{p}$. 1171.

${ }^{6}$ Cf. également Guillermo Sheridan, « Gilberto Owen y Federico García Lorca viajan a la luna », p. 21.

R. B. E. C. T., vol 6, núm. 1, jan-abr.2013 ISSN - 1982-873X

Artigo apresentado na Premières Journées d'études organisées à Lyon (France) 
Dans les deux poèmes le substantif guarismo fait référence au chiffre 8 et c'est justement le sens figuratif qu'il donne à ce substantif qui confirme cette lecture. Dans « El río sin tacto » les guarismos sont des traces de pas sur le sable de la plage ; dans " Nueva nao " il est question de " la huella de tu paso ». En effet, le chiffre 8, semblable -nous le rappelons- à des traces de pieds sur le sol ou sur le sable permet le rapprochement. Bref, il està la fois, dans les deux poèmes, image et chiffre.

Enfin, la valeur symbolique du chiffre 8 chez Owen ne se limite pas à sa valeur iconographique et se nourrit, une fois de plus, des mathématiques. En effet, le symbole mathématiquereprésentant l'infini n'est autre qu'un 8 couché, $\infty$, introduit en mathématiques par le mathématicien anglais John Walis et dont la forme rappelle le ruban de Möbius, rejoignant ainsi le symbolisme de l'Ouroboros ou serpent qui se mord la queue, qui évoque l'infini, l'éternel retour $^{1}$. Ce symbole, de par ses caractéristiques morphologiques et son pouvoir visuel d'évocation, pourrait donc évoquer chez Owen l'infini, comme le suggèrele poème 8 de Nueva Nao de Amor, où le verbe repite et l'adjectif interminable rejoignent ainsi le symbolisme du $\infty$, dont il est question également dans ce poème« Guarismo que repite, interminable, la huella de tu paso ».

Pour conclure, l'œuvre d'Owen constitue un exemple assez original d'utilisation des mathématiques en poésie, et cet emploi s'étend même à ses romans et écrits personnels. Le chiffre ou plus généralement le symbole mathématique devient tour à tour dessin, signe linguistique, dans une étrange réversibilité qui ouvre à toutes les possibilités significatives et expressives du signe. Au fond, semble dire Owen renouant avec les Pythagoriciens, les mathématiques et les arts (poésie, musique) ne sont pas des univers aussi étrangers qu'ils en ont l'air. Owen, rejoignant ainsiles Pythagoriciens, révèle les liens entre les mathématiques et la poésie, liens qu'il pousse à l'extrême lorsque chiffres et lettres deviennent réversibles, comme si on pouvait voir simultanément, en trompe-l'œil, deux signes différents qui se superposent, un peu à la façon de l'image hybride de la lampe-ange de Lautréamont ${ }^{2}$. Ce faisant, Owen nous rappelle que tout matériau peut accéder au statut poétique, révélant une conception très

${ }^{1}$ « L'Ouroboros lui aussi est symbole de manifestation et de résorption cyclique [...] selon les termes de Bachelard, il est la dialectique matériellede la vie et de la mort, la mort qui sort de la vie et la vie qui sort de la mort. S'il appelle l'image du cercle, il est surtout la dynamique du cercle, c'est-à-dire la première roue, d'apparence immobile, parce qu'elle ne tourne que sur elle-même, mais dont le mouvement est infini, parce qu'il se reconduit perpétuellement en lui-même ». Jean Chevalier et Alain Gheerbrant, Dictionnaire des symboles, article "Serpent ", p. 868. Ce sont les auteurs qui soulignent.

${ }^{2}$ « Une lampe et un ange qui forment un même corps, voilà ce que l’on ne voit pas souvent. II reconnaît la forme de la lampe, il reconnaît la forme de l'ange ; mais il ne peut pas les scinder dans son esprit ", Lautréamont, ibid., p. 96. 
moderne de la poésie. II met en évidence, en outre, les rapports plus étroits que l'on ne le croit entre les chiffres et les lettres, rappelant l'image de Lacan : « Les astronautes [...] vont dans la lune portés par un écrit », car c'est la lettre, comme le signale Brigitte Compain, ou plutôt " l'écriture mathématique de l'univers (formule de Newton qui se réduit à une suite de lettres) qui permet de construire le lemme qui va transporter l'astronaute dans l'espace ${ }^{1}$. Si les lettres servent à élaborer de complexes formules mathématiques, on peut faire également de la poésie à l'aide de chiffres.C'est peut-être pour cela que pour Owen le chiffre constitue, à lui seul, un poème, autosuffisant en définitive, dans son halo de beauté et d'abstraction : «Pero el número, te digo, es nomás un poema. Nada más. Una teoría de una teoría fantasma de fantasma»².

\section{Bibliographie}

Allaigre-Duny, Annick, « La passion de l'équilibre » et Brigitte Compain, " Cuesta chimiste écrivant ", in Annick Allaigre-Duny (éd.), Jorge Cuesta. Littérature, histoire, psychanalyse, Paris, L'Harmattan, 2006.

Arredondo, Inés, "Apuntes para una biografía ", Revista de Bellas Artes, Tercera época, noviembre 1982, pp. 43-48.

Bernard, Suzanne, Le poème en prose, de Baudelaire jusqu'à nos jours, Paris, Nizet, 1994.

Darío, Rubén, Azul... / Cantos de Vida y Esperanza, Edición de Alvaro Alonso, coll. Austral, Madrid, Espasa Calpe, 1992.

de la Motte, Annette, Au-delà du mot. Une « écriture du silence » dans la littérature française au vingtième siècle, Ars Rhetorica 14, Lit Verlag Münster, 2004.

Ducasse, Isidore, Comte de Lautréamont, CEuvres Complètes, Edition d'Hubert Juin, Poésie/Gallimard, Paris, Gallimard, 1973, réimpression de 1993.

Gómez de la Serna, Ramón, Greguerías, edición de Rodolfo Cardona, Madrid, Cátedra, Letras Hispánicas, 1a edición 1980, 13a edición 2006

Herrera, Hayden, Frida, Biographie de Frida Kahlo, Paris, Ed. Anne Carrière, 1996 pour la traduction française.

Meusnier, Norbert, "Isidore Ducasse, géomètre de la poésie ", Alliage, $n^{\circ}$ 57-58, http://www.tribunes.com/tribune/alliage/57/Meusnier.htm

Otaola González, Paloma, "En torno a la música y las artes liberales », in Nassarre, Revista aragonesa de musicología IX, 1, Institución Fernando el Católico, Zaragoza, 1993, p. 145-180.

\footnotetext{
${ }^{1}$ Brigitte Compain, « Cuesta chimiste écrivant », p. 286.

${ }^{2}$ P. 271. Voir traduction à la note 25.
}

R. B. E. C. T., vol 6, núm. 1, jan-abr.2013 ISSN - 1982-873X 
Owen, Gilberto, Obras, México, Fondo de Cultura Económica, 1979, réimpression de 1996.

Pierssens, Michel, Ducasse et Lautréamont : L'envers et l'endroit, Paris, Editions des Presses Universitaires de Vincennes et Du Lérot, éditeur, 2005.

Sheridan, Guillermo, "Gilberto Owen y el torbellino rubio ", in Vuelta, $n^{\circ} 239$, octubre de 1996, pp. 6-12, http://www.letraslibres.com/pdf.php?id=4904

- "Gilberto Owen y Federico García Lorca viajan a la luna ", in Vuelta, n²58, mayo de 1998, pp. 16-21.

Stubhaug, Arild, Sophus Lie : une pensée audacieuse, Paris ; Berlin ; Heidelberg, Springer, 2005.

\section{DICTIONNAIRES :}

Chevalier, Jean et Gheerbrant, Alain, Dictionnaire des symboles, Paris, Robert Laffont / Jupiter, Ed. de 1982. Articles consultés ou cités : « Sept », p. 860 ; « Serpent », p. 868.

Diccionario de la Real Academia de la Lengua Española, 22 e édition, Madrid, Espasa Calpe, 2001. Entrées consultées ou citées : "Cifra », 2e tome, p. 551 ; " Guarismo », 1er tome, p. 1171 ; « Neuma», 2e tome, p. 1578.

Le Littré, http://francois.gannaz.free.fr/Littre/accueil.php. Entrées consultées ou citées : « Chiffe ", http://francois.gannaz.free.fr/Littre/xmlittre.php?requete=chiffre\&submit=Rechercher ; « Neume",

http://francois.gannaz.free.fr/Littre/xmlittre.php?requete=neume\&submit=Rechercher ; "X ", http://francois.gannaz.free.fr/Littre/xmlittre.php?rand=\&requete=x\&submit=Rechercher Le Petit Robert, édition revue, corrigée et mise à jour pour 1989. Paris, Le Robert, 1989. Entrées consultées ou citées : " chiffre », p. 305. 